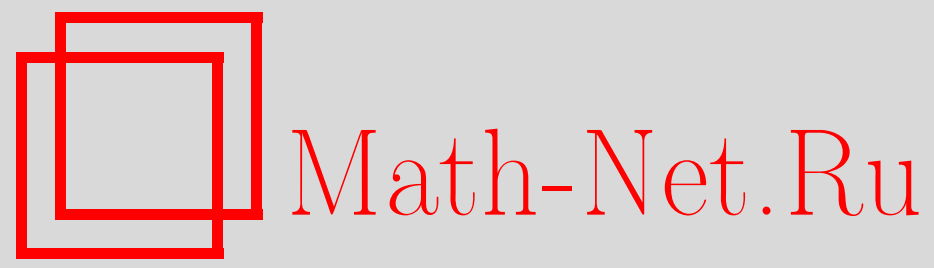

А. Т. Усс, О краевых задачах для четырехмерных аналогов системы КошиРимана с комплексными коэффициентами, Вестн. Сам. гос. техн. ун-та. Сер. Физ.-мат. науки, 2001, выпуск 12, 10-16

DOI: https://doi.org/10.14498/vsgtu55

Использование Общероссийского математического портала Math-Net.Ru подразумевает, что вы прочитали и согласны с пользовательским соглашением

http: //www.mathnet.ru/rus/agreement

Параметры загрузки:

IP : 35.174 .16 .151

26 апреля 2023 г., $12: 15: 36$ 


\section{A.T. Уcc}

\section{О КРАЕВЫХ ЗАДАЧАХ ДЛЯ ЧЕТЫРЕХМЕРНЫХ АНАЛОГОВ СИСТЕМЫ КОШИ-РИМАНА С КОМПЛЕКСНЫМИ КОЭФФИЦИЕНТАМИ}

Проведена гомотопическая классификация эллиптических систем двух дифференциальных уравнений первого порядка с комплексными коэффициентами, являющихся обобщением на четырехмерный случай известной системы Коши-Римана. Доказывается, что системы указанного типа не имеют ни одной регуляризуемой краевой задачи ни в какой ограниченной области в $R^{4}$.

Одним из способов построения аналога голоморфных функций комплексной переменной в многомерном случае является обобщение условий Коши-Римана. На сегодняшний день известно много таких обобщений ( см. [1-4] и имеющиеся там ссылки ), и они эффективно используются в теории краевых задач для систем дифференциальных уравнений, например, [5 - 7]. В настоящей работе вводится класс систем дифференциальных уравнений, содержащий не изучавшиеся ранее аналоги систем Коши-Римана в четырехмерном пространстве, исследуется топологическое строение этого класса и возможность постановки нетеровых краевых задач для его представителей.

В $R^{4}$ рассмотрим систему двух дифференциальных уравнений вида

$$
\sum_{j=1}^{4} A_{j} \frac{\partial U}{\partial x^{j}}=0,
$$

где $A_{j}(j=1,2,3,4)$ - постоянные комплексные матрицы размера $2 \times 2, U=(u, v)^{\mathrm{T}}-$ искомый вектор-столбец из комплекснозначных функций $u=u(x)$ и $v=v(x) ; x=\left(x^{1}, x^{2}, x^{3}, x^{4}\right) \in \nabla^{4}$. Следуя [8] (с. 258), назовем систему (1) четырехмерным аналогом системы Коши-Римана ( сокращенно, ЧКР-системой ), если компоненты $u$ и $v$ каждого ее решения $U$ являются ( комплексными ) гармоническими функциями.

Анализ рассуждений, проведенных в [3] для систем дифференциальных уравнений первого порядка в $\nabla^{n}$ с действительными коэффициентами, показывает их применимость и в случае комплексных коэффициентов. Поэтому заключаем, что каждая ЧКР-система эллиптична, и произвольная система вида (1) является ЧКР в том и только в том случае, когда матрицы $A_{j}$ $(j=1,2,3,4)$ обратимы и удовлетворяют равенствам

$$
A_{j}^{-1} A_{k}+A_{k}^{-1} A_{j}=2 \delta_{j k} E \quad(j, k=1,2,3,4),
$$

где $\delta_{j k}-$ символ Кронекера, а $E$ - единичная матрица второго порядка.

Умножением, в случае необходимости, эллиптической системы (1) на матрицу $A_{1}^{-1}$ и переобозначением матричных коэффициентов в получающейся при этом системе можно добиться того, что матрица $A_{1}$ в системе (1) будет единичной. Поэтому впредь будем предполагать, если не оговорено противное, что в ЧКР-системе (1) $A_{1}=E$.

Пусть система (1) является ЧКР. Тогда из равенств (2) и равенства $A_{1}=E$ следует, что матрицы $A_{2}, A_{3}$ и $A_{4}$ обладают свойством:

$$
A_{j}+A_{j}^{-1}=0 \quad(j=2,3,4) .
$$

Из этих равенств заключаем, что $\operatorname{det} A_{j}= \pm 1 \quad(j=2,3,4)$.

Покажем, что ни одно из равенств $\operatorname{det} A_{j}=-1 \quad(j=2,3,4)$ не может иметь места. Убедимся в этом на примере матрицы $A_{2}$, для остальных матриц рассуждения проводятся аналогично.

Предположим, что $\operatorname{det} A_{2}=-1$. Тогда из равенства $A_{2}+A_{2}^{-1}=0$ следует, что матрица $A_{2}$ необходимо должна совпадать с одной из матриц

$$
\left(\begin{array}{ll}
i & 0 \\
0 & i
\end{array}\right),\left(\begin{array}{cc}
-i & 0 \\
0 & -i
\end{array}\right)
$$

Далее, определитель матрицы $A_{3}$ равен либо 1, либо -1. Но -1 он равняться не может, так как в противном случае матрица $A_{3}$ также должна находиться среди матриц (3), а для матриц этого 
вида равенство $A_{2}^{-1} A_{3}+A_{3}^{-1} A_{2}=0$ не выполняется. Значит, det $A_{3}=-1$. Отсюда, а также из равенства $A_{3}+A_{3}^{-1}=0$ следует, что

$$
A_{3}=\left(\begin{array}{rr}
a & b \\
c & -a
\end{array}\right),
$$

где комплексные числа $a, b$ и $c$ - таковы, что $a^{2}+b c=-1$. Это, вместе с соотношением $A_{2}^{-1} A_{3}+A_{3}^{-1} A_{2}=0$, приводит нас к равенству

$$
\left(\begin{array}{cc}
i a & i b \\
i c & -i a
\end{array}\right)=0,
$$

т. е. $A_{3}=0$, что противоречит обратимости матрицы $A_{3}$.

Итак, равенство $\operatorname{det} A_{j}=-1$ не может иметь места, и, следовательно, $\operatorname{det} A_{j}=1$ при $j=2$, 3,4 .

Л е м м а. Пусть в ЧКР-системе (1) матрица $A_{1}-$ единичная. Тогда матрица $A_{2}$ подобна матрице

$$
B_{2}=\left(\begin{array}{rr}
i & 0 \\
0 & -i
\end{array}\right),
$$

m.е. существует такая невырожденная матрица $T$ второго порядка, что $B_{2}=T A_{2} T^{-1}$.

Д о к а з а т е л ь с т в о. Жорданова нормальная форма над полем С произвольной квадратной матрицы второго порядка есть одна из матриц

$$
\left(\begin{array}{ll}
\lambda & 1 \\
0 & \lambda
\end{array}\right),\left(\begin{array}{ll}
\lambda & 0 \\
1 & \lambda
\end{array}\right),\left(\begin{array}{cc}
\lambda_{1} & 0 \\
0 & \lambda_{2}
\end{array}\right),
$$

где $\lambda, \lambda_{1}, \lambda_{2}$ - некоторые комплексные числа. C другой стороны, каждая матрица $B$, подобная матрице $A_{2}$, должна удовлетворять равенству $B+B^{-1}=0$. Поскольку последнему равенству из трех выписанных матриц может удовлетворять лишь диагональная матрица и лишь в случае, когда

$$
\lambda_{1}^{2}=\lambda_{2}^{2}=-1
$$

а матрицы $B_{2}$ и - $B_{2}$ - подобны, то заключаем, что матрица $A_{2}$ подобна матрице $B_{2}$. Лемма доказана.

Пусть теперь $T$ - матрица, указанная в лемме. Тогда, умножая ЧКР-систему (1) на матрицу $T$ и меняя в системе искомую функцию $U$ на $V=T U$, мы приходим к ЧКР-системе

$$
\sum_{j=1}^{4} B_{j} \frac{\partial V}{\partial x^{j}}=0
$$

в которой матрица $B_{1}$ - единичная, a $B_{2}$ - матрица, указанная в лемме. Поскольку система (4) имеет тип ЧКР, матрицы $B_{3}$ и $B_{4}$ не могут быть произвольными. Как показывает простой анализ соотношений (2), они необходимо должны иметь вид:

$$
B_{3}=\left(\begin{array}{cc}
0 & b \\
-b^{-1} & 0
\end{array}\right), \quad B_{4}=\left(\begin{array}{cc}
0 & c \\
-c^{-1} & 0
\end{array}\right),
$$

где $b$ и $c$-ненулевые комплексные числа, связанные равенством $b^{2}+c^{2}=0$. Таким образом, мы приходим к следующему заключению.

Т е о р е м а 1. Если (1) является ЧКР-системой, то существует число $b \in \mathfrak{R} \backslash\{0\} \quad u \quad \mathfrak{R}-$ линейное невырожденное преобразование $T: \Re^{2} \rightarrow \Re^{2}$ такие, что система (1) равносильна

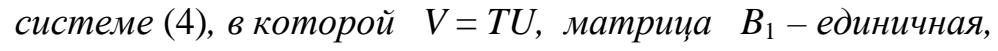

$$
B_{2}=\left(\begin{array}{cc}
i & 0 \\
0 & -i
\end{array}\right), B_{3}=\left(\begin{array}{cc}
0 & b \\
-b^{-1} & 0
\end{array}\right),
$$

а матрица $B_{4}$ - одна из следующих двух: 


$$
\left(\begin{array}{cc}
0 & i b \\
i b^{-1} & 0
\end{array}\right),\left(\begin{array}{cc}
0 & -i b \\
-i b^{-1} & 0
\end{array}\right)
$$

При каждом $b \in \mathfrak{R} \backslash\{0\}$ и матрицах (5), (6) система (4) является ЧКР-системой.

Проведем гомотопическую классификацию ЧКР-систем.

Назовем две ЧКР-системы вида (1) гомотопными, если они гомотопны в классе эллиптических систем двух дифференциальных уравнений первого порядка с постоянными комплексными коэффициентами, причем гомотопирующее семейство, связывающее эти системы, можно выбрать таким образом, что каждый его элемент является ЧКР-системой.

Т е о р е м а 2. Множество ЧКР-систем имеет две компоненты гомотопической связности, и каждая ЧКР-система (1) гомотопна системе, характеристическая матрица которой есть одна из двух следующих:

$$
\left(\begin{array}{cc}
\xi_{1}+i \xi_{2} & \xi_{3} \pm i \xi_{4} \\
-\xi_{3} \pm i \xi_{4} & \xi_{1}+i \xi_{2}
\end{array}\right) .
$$

Д о к а з а т е л ь с т в о. Покажем сначала, что характеристическая матрица произвольной ЧКР-системы (1) в классе характеристических матриц ЧКР-систем может быть сгомотопирована к одной из матриц (7).

Пусть (1) - произвольная система типа ЧКР ( равенство $A_{1}=E$ не предполагается ). Тогда характеристическую матрицу этой системы, обозначим ее $A(\xi)$, можно представить в виде:

$$
A(\xi)=A_{1}\left(E \xi_{1}+A_{1}^{-1} A_{2} \xi_{2}+A_{1}^{-1} A_{3} \xi_{3}+A_{1}^{-1} A_{4} \xi_{4}\right) .
$$

Согласно доказанной выше лемме существует такая невырожденная матрица второго порядка $T$, что $T A_{1}^{-1} A_{2} T^{-1}=B_{2}$, где $B_{2}-$ первая из матриц (5). Следовательно,

$$
A(\xi)=A_{1} T^{-1}\left(E \xi_{1}+B_{2} \xi_{2}+B_{3} \xi_{3}+B_{4} \xi_{4}\right) T,
$$

где при некотором $b \in \Re \backslash\{0\}$ матрицы $B_{2}$ и $B_{3}$ задаются равенствами (5), а $B_{4}$ - одна из матриц (6). Матрицы $A_{1}$ и $T$-невырожденные и, в силу связности группы комплексных невырожденных матриц ( см., например, [9], с.28 и 56 ), гомотопны единичной матрице $E$. Обозначим через $A_{1}(t)$ и $T(t), 0 \leq t \leq 1$, пути в группе указанных матриц с началами, соответственно, в $A_{1}$ и в $T$ и с концами в $E$, и положим:

$$
\begin{gathered}
b(t)=(t+(1-t)|b|) \exp \{i(1-t) \arg b\}, \\
B_{3}(t)=\left(\begin{array}{cc}
0 & b(t) \\
-b^{-1}(t) & 0
\end{array}\right), \\
B_{4}(t)=\left(\begin{array}{cc}
0 & i b(t) \\
i b^{-1}(t) & 0
\end{array}\right), \text { если } B_{4}-\text { первая из матриц (6), и } \\
B_{4}(t)=\left(\begin{array}{cc}
0 & -i b(t) \\
-i b^{-1}(t) & 0
\end{array}\right), \text { если } B_{4}-\text { вторая из матриц (6). }
\end{gathered}
$$

Тогда гомотопия

$$
A(t, \xi)=A_{1}(t)(T(t))^{-1}\left(E \xi_{1}+B_{2} \xi_{2}+B_{3}(t) \xi_{3}+B_{4}(t) \xi_{4}\right) T(t),
$$

$0 \leq t \leq 1$, связывает матрицу $A(\xi)$ с одной из матриц (7). При этом, очевидно, при каждом $t \in[0 ; 1]$ матрица $A(t, \xi)$ является характеристической матрицей некоторой ЧКР-системы.

Докажем теперь негомотопность матриц

$$
\left(\begin{array}{cc}
\xi_{1}+i \xi_{2} & \xi_{3}+i \xi_{4} \\
-\xi_{3}+i \xi_{4} & \xi_{1}+i \xi_{2}
\end{array}\right),\left(\begin{array}{cc}
\xi_{1}+i \xi_{2} & \xi_{3}-i \xi_{4} \\
-\xi_{3}-i \xi_{4} & \xi_{1}+i \xi_{2}
\end{array}\right) .
$$

на множестве полиномиальных матриц, невырожденных при $\left(\xi_{1}, \xi_{2}, \xi_{3}, \xi_{4}\right) \in \nabla^{4} \backslash\{0\}$.

Предположим противное, т.е. предположим, что выписанные матрицы гомотопны на указанном множестве. Тогда гомотопными будут и векторные поля $l_{1}, l_{2}: S^{3} \rightarrow S^{3}$, определяемые 
на единичной сфере $S^{3}:=\left\{\xi=\left(\xi_{1}, \xi_{2}, \xi_{3}, \xi_{4}\right) \in \mathrm{R}^{4} \mid \xi_{1}^{2}+\xi_{2}^{2}+\xi_{3}^{2}+\xi_{4}^{2}=1\right\}$ первыми строками этих матриц:

$$
l_{1}(\xi)=\xi, \quad l_{2}(\xi)=\left(\xi_{1}, \xi_{2}, \xi_{3},-\xi_{4}\right) .
$$

Следовательно, должны совпадать ( см. [10], с. 429 ) степени отображений $l_{1}$ и $l_{2}$. Но для первого отображения степень равна единице, а для второго - минус единице. Противоречие.

Таким образом, каждая ЧКР-система (1) может быть сгомотопирована в классе ЧКР-систем к одной из двух негомотопных между собой систем, описываемых характеристическими матрицами (7). Теорема доказана.

3 а м е ч а н и е 1. Гомотопическая классификация общих эллиптических систем двух дифференциальных уравнений вида (1) ранее проведена В.И. Шевченко [11] ( см. также [12] ), и, как показывает сравнение результатов [11] с утверждением теоремы 2, рассмотренное здесь сужение класса систем дифференциальных уравнений, а также сужение класса допустимых гомотопий не изменяет число компонент гомотопической связности.

3 а м е ч а н и е 2. Рассуждения, проведенные в доказательстве теоремы 2, показывают, что степень отображения $\rho: S^{3} \rightarrow \nabla^{4} \backslash\{0\}$, задаваемого первой строкой характеристической матрицы

$$
A(\xi)=\left(\begin{array}{ll}
a_{1}(\xi) & a_{2}(\xi) \\
a_{3}(\xi) & a_{4}(\xi)
\end{array}\right)
$$

системы (1) равенством

$$
\rho(\xi)=\left(\operatorname{Re} a_{1}(\xi), \operatorname{Im} a_{1}(\xi), \operatorname{Re} a_{2}(\xi), \operatorname{Im} a_{2}(\xi)\right), \quad \xi \in S^{3},
$$

является инвариантом, различающим компоненты связности множества всех ЧКР-систем и, более того, множества всех эллиптических систем двух дифференциальных уравнений первого порядка с комплексными коэффициентами в $\mathrm{R}^{4}$. Поскольку же $a_{1}(\xi)$ и $a_{2}(\xi)$ - линейные формы относительно переменных $\xi_{1}, \ldots, \xi_{4}$, то отображение $\rho$ есть сужение на $S^{3}$ линейного невырожденного ( в силу эллиптичности системы (1) ) отображения из $\mathrm{R}^{4}$ в $\mathrm{R}^{4}$, а потому его степень равна знаку определителя последнего отображения ( см., например, теорему 6.1 в [13], с. 24 ). Таким образом, мы получаем простой алгебраический признак различия компонент гомотопической связности указанных выше множеств систем дифференциальных уравнений ( ср. с [12] ): две системы вида (1) гомотопны тогда и только тогда, когда имеют одинаковые знаки определители линейных отображений, построенных описанным способом по характеристическим матрицам этих систем.

Перейдем к рассмотрению краевых задач для ЧКР-систем. Без ограничения общности будем предполагать, что ЧКР-система (1) имеет канонический вид (4), а именно, $A_{1}=B_{1}=E$, матрицы $A_{2}=B_{2}$ и $A_{3}=B_{3}$ определяются равенствами (5), а $A_{4}=B_{4}$ - первая из матриц (6) (случай второй матрицы из (6) сводится к рассматриваемому простой заменой переменных в $\nabla^{4}$ ). Пусть, далее, $\Omega$ - ограниченная область в $\mathrm{R}^{4}$, границей которой является бесконечно гладкое трехмерное многообразие $\partial \Omega$.

Комплексное граничное условие на $\partial \Omega$ для решения $U=(u, v)^{\mathrm{T}}$ системы (1), рассматриваемой в области $\Omega$, не приводит к краевой задаче самого общего вида, поскольку порядки действительной или мнимой частей комплексного оператора в общем случае не обязаны совпадать с порядком самого оператора. Постановка же действительных граничных условий для решений системы (1) делает целесообразной замену системы (1) на эквивалентную ей систему четырех дифференциальных уравнений первого порядка с действительными коэффициентами. Условимся в дальнейшем обозначать через $U$ вектор-столбец $\left(u_{1}, u_{2}, u_{3}, u_{4}\right)^{\mathrm{T}}$, составленный из действительных и мнимых частей компонент решения $U$ исходной системы $(1): u_{1}=\operatorname{Re} u$, $u_{2}=\operatorname{Im} u, u_{3}=\operatorname{Re} v, u_{4}=\operatorname{Im} v$. Тогда овеществленный вариант ЧКР-системы (1) канонического вида можно записать в том же виде (1), только теперь уже матрица $A_{1}$ будет единичной матрицей четвертого порядка, и

$$
A_{2}=\left(\begin{array}{cccc}
0 & 1 & 0 & 0 \\
-1 & 0 & 0 & 0 \\
0 & 0 & 0 & -1 \\
0 & 0 & 1 & 0
\end{array}\right), \quad A_{3}=\left(\begin{array}{cccc}
0 & 0 & b_{1} & -b_{2} \\
0 & 0 & b_{2} & b_{1} \\
-k b_{1} & -k b_{2} & 0 & 0 \\
k b_{2} & -k b_{1} & 0 & 0
\end{array}\right), A_{4}=\left(\begin{array}{cccc}
0 & 0 & -b_{2} & -b_{1} \\
0 & 0 & b_{1} & -b_{2} \\
k b_{2} & -k b_{1} & 0 & 0 \\
k b_{1} & k b_{2} & 0 & 0
\end{array}\right) .
$$


Здесь $b_{1}=\operatorname{Re} b, b_{2}=\operatorname{Im} b$ и $k=\left(b_{1}^{2}+b_{2}^{2}\right)^{-1}$, где $b-$ число, входящее в матрицы (5), (6).

Итак, рассмотрим краевую задачу отыскания решения $U=\left(u_{1}, u_{2}, u_{3}, u_{4}\right)^{\mathrm{T}}$ эллиптической системы четырех дифференциальных уравнений

$$
\mathrm{A}\left(\frac{\partial}{\partial x}\right) U:=\sum_{j=1}^{4} A_{j} \frac{\partial U}{\partial x^{j}}=f(x), \quad x=\left(x^{1}, x^{2}, x^{3}, x^{4}\right) \in \Omega,
$$

удовлетворяющего граничным условиям

$$
\left.\mathrm{B}\left(y, \frac{\partial}{\partial x}\right)\right|_{\Omega \ni x \rightarrow y} U=g(y), \quad y \in \partial \Omega .
$$

Здесь $A_{1}=E_{4}$ - единичная матрица четвертого порядка, а матрицы $A_{2}, A_{3}$ и $A_{4}$ задаются равенствами (8), в которых $b_{1}$ и $b_{2}$ - произвольные действительные числа, удовлетворяющие условию $b_{1}^{2}+b_{2}^{2}>0$, и $k=\left(b_{1}^{2}+b_{2}^{2}\right)^{-1} ; f$ и $g$ - заданные соответственно четырех- и двухкомпонентный вектор-столбцы, состоящие из действительных функций; В | матричный, размера $2 \times 4$, граничный оператор, состоящий из скалярных линейных операторов, «полиномиальных относительно нормали» к $\partial \Omega$ [14].

Краевая задача называется регуляризуемой, если для нее выполнено условие Я.Б. Лопатинского [15]. Известно $[14,16]$, что регуляризуемость краевой задачи есть необходимое и достаточное условие нетеровости оператора, отвечающего этой задаче и действующего в определенных банаховых пространствах.

Т е о р е м а 3. Краевая задача (9), (10) не является регуляризуемой.

Д о к а з а т е л ь с т в о. Достаточно установить невыполненность условия Лопатинского для краевой задачи в полупространстве $\mathrm{R}_{+}^{4}:=\left\{x=\left(x^{1}, x^{2}, x^{3}, x^{4}\right) \in \nabla^{4} \mid x^{1}>0\right\}$, получаемой из задачи (9), (10) «замораживанием коэффициентов» в той точке многообразия $\partial \Omega$, в которой нормаль к $\partial \Omega$ параллельна вектору $(1,0,0,0)$. Поэтому при доказательстве теоремы можно считать, что $\Omega=\mathrm{R}_{+}^{4}$ и символ главной части граничного оператора $\mathrm{B} \mid x^{1}=0$ не зависит от точки $y \in \partial \Omega$. Далее, поскольку система (9) позволяет выразить $\frac{\partial U}{\partial x^{1}}$ через частные производные от $U$ по другим переменным, без ограничения общности можно предполагать, что граничные условия (10) не содержат дифференцирования по $x^{1}$. Пусть

$$
A(\lambda, \tau)=A_{1} \lambda+A_{2} \tau_{1}+A_{3} \tau_{2}+A_{4} \tau_{3}
$$

есть характеристическая матрица системы (9), а $B(\tau)$ - символ главной части оператора $\left.\mathrm{B}\left(-i \frac{\partial}{\partial x}\right)\right|_{x^{1}=0}$. Тогда для доказательства теоремы нам остается доказать, что существует ненулевой набор $\tau=\left(\tau_{1}, \tau_{2}, \tau_{3}\right) \in \nabla^{3} \backslash\{0\}$, при котором ранг матрицы

$$
(2 \pi i)^{-1} B(\tau) \cdot \int_{\gamma} A^{-1}(\lambda, \tau) d \lambda
$$

строго меньше двух ( здесь $\gamma$ - простой гладкий замкнутый контур, лежащий в верхней $\lambda-$ полуплоскости и охватывающий все находящиеся в ней $\lambda$-корни уравнения $\operatorname{det} A(\lambda, \tau)=0)$.

Матрица $B(\tau)$ представляет собой $2 \times 4$-матрицу, элементами которой являются действительнозначные непрерывные однородные функции переменных $\tau_{1}, \tau_{2}, \tau_{3}$. Будем предполагать, что ее ранг равен двум при каждом $\tau \in \mathrm{R}^{3} \backslash\{0\}$, поскольку в противном случае ранг матрицы (11) заведомо меньше двух хотя бы при одном $\tau \neq 0$. Обозначим через $\Lambda_{j k}$ и $H_{j k}(j, k=1,2,3$, 4) миноры второго порядка, составленные из $j$-ых и $k$-ых столбцов соответственно матрицы $B(\tau)$ и матрицы (11), и положим:

$$
L_{1}:=-\Lambda_{12}+k \Lambda_{34}, L_{1}:=\Lambda_{13}+\Lambda_{24}, L_{3}:=\Lambda_{14}-\Lambda_{23}, \beta_{1}:=-b_{1} \tau_{2}+b_{2} \tau_{3}, \beta_{2}:=b_{2} \tau_{2}+b_{1} \tau_{3} .
$$

Тогда непосредственным вычислением миноров $H_{j k}(1 \leq j<k \leq 4)$ матрицы (11) получим, что в каждой точке $\tau=\left(\tau_{1}, \tau_{2}, \tau_{3}\right)$ единичной сферы $S^{2}=\left\{\xi=\left(\xi_{1}, \xi_{2}, \xi_{3}\right) \in \mathrm{R}^{3} \mid \xi_{1}^{2}+\xi_{2}^{2}+\xi_{3}^{2}=1\right\}$

$$
H_{12}=\left(1-\tau_{1}^{2}\right) L_{1}-k \tau_{1} \beta_{1} L_{2}-k \tau_{1} \beta_{2} L_{3}+i k\left(\beta_{2} L_{2}-\beta_{1} L_{3}\right) \text {, }
$$




$$
\begin{gathered}
H_{13}=\tau_{1}\left(\beta_{1} L_{1}-\tau_{2} L_{2}\right)-k \beta_{2}\left(\beta_{2} L_{2}-\beta_{1} L_{3}\right)+i\left(\beta_{2} L_{1}-\tau_{1} L_{3}\right), \\
H_{14}=\tau_{1}\left(\beta_{2} L_{1}-\tau_{2} L_{3}\right)+k \beta_{1}\left(\beta_{2} L_{2}-\beta_{1} L_{3}\right)-i\left(\beta_{1} L_{1}-\tau_{1} L_{2}\right), \\
H_{23}=-H_{14}, \quad H_{24}=H_{13}, \quad H_{34}=-k^{-1} H_{12} .
\end{gathered}
$$

Зададим непрерывное отображение $\varphi: S^{2} \rightarrow S^{2}$ равенством

$$
\varphi(\tau)=\left(\tau_{1},-b_{1} \tau_{2}+b_{2} \tau_{3}, b_{2} \tau_{2}+b_{1} \tau_{3}\right) / l\left(\tau_{1}\right), \quad \tau=\left(\tau_{1}, \tau_{2}, \tau_{3}\right) \in S^{2},
$$

где $l\left(\tau_{1}\right)=\left(\tau_{1}^{2}+\left(b_{1}^{2}+b_{2}^{2}\right)\left(1-\tau_{1}^{2}\right)\right)^{1 / 2}$. Поскольку, как нетрудно убедиться, система уравнений относительно $\tau_{1}, \tau_{2}, \tau_{3}$

$$
\xi_{1}=\tau_{1} / l\left(\tau_{1}\right), \quad \xi_{2}=\left(-b_{1} \tau_{2}+b_{2} \tau_{3}\right) / l\left(\tau_{1}\right), \quad \xi_{3}=\left(b_{2} \tau_{2}+b_{1} \tau_{3}\right) / l\left(\tau_{1}\right)
$$

однозначно разрешима при каждом $\xi_{,}=\left(\xi_{1}, \xi_{2}, \xi_{3}\right) \in S^{2}$ и решение ее

$$
\tau_{1}=\xi_{1} m\left(\xi_{1}\right), \quad \tau_{2}=\left(-b_{1} \xi_{2}+b_{2} \xi_{3}\right) k m\left(\xi_{1}\right), \quad \tau_{3}=\left(b_{2} \xi_{2}+b_{1} \xi_{3}\right) k m\left(\xi_{1}\right)
$$

где $m\left(\xi_{1}\right)=\left(\left(b_{1}^{2}+b_{2}^{2}\right) /\left(1-\xi_{1}^{2}+\left(b_{1}^{2}+b_{2}^{2}\right) \xi_{1}^{2}\right)\right)^{1 / 2}$, образует точку $\tau=\left(\tau_{1}, \tau_{2}, \tau_{3}\right)$, принадлежащую сфере $S^{2}$, то заключаем, что отображение $\varphi$ является гомеоморфизмом сферы $S^{2}$ на себя.

Так как ранг матрицы $B(\tau)$ при каждом $\tau \in \mathrm{R}^{3} \backslash\{0\}$ равен двум, то в каждой точке $\tau \in S^{2}$ определен ненулевой вектор $L(\tau)=\left(L_{1}, L_{2}, L_{3}\right)$. Значит, на двумерной сфере $S^{2}$ определено непрерывное невырождающееся векторное поле $L L \varphi^{-1}$. Согласно теореме «о еже» ( см., например, [10], с. 584 ) на сфере $S^{2}$ найдется точка $\varsigma \in S^{2}$ такая, что

$$
\left(L\left\llcorner\varphi^{-1}\right)(\varsigma)=\alpha \varsigma,\right.
$$

где $\alpha-$ некоторое действительное число. Последнее равенство показывает, что в точке $\tau=\varphi^{-1}(\varsigma) \in S^{2}$ имеют место равенства:

$$
L_{1}=\alpha \tau_{1} / l\left(\tau_{1}\right), \quad L_{2}=\alpha\left(-b_{1} \tau_{2}+b_{2} \tau_{3}\right) / l\left(\tau_{1}\right), \quad L_{3}=\alpha\left(b_{2} \tau_{2}+b_{1} \tau_{3}\right) / l\left(\tau_{1}\right) .
$$

Подставляя найденные выражения для $L_{1}, L_{2}$ и $L_{3}$ в указанные выше миноры матрицы (11), получим, что в точке $\tau=\varphi^{-1}(\varsigma) \in S^{2}$

$$
H_{12}=H_{12}=H_{14}=H_{23}=H_{24}=H_{34}=0 .
$$

Таким образом, в точке $\tau=\varphi^{-1}(\varsigma) \in S^{2}$ ранг матрицы (11) строго меньше двух. Теорема доказана.

Из теоремы 3 непосредственно выводим

С л е д с т в и е. Для ЧКР-системь (1), рассматриваемой в ограниченной области $\Omega \subset \nabla^{4}$, нет псевдодифференциальных граничных условий, «полиномиальных относительно нормали», образуюших с этой системой регуляризуемую краевую задачу.

3 а м е ч а н и е 3. Для частного случая ЧКР-системы (1), а именно, в случае, когда характеристическая матрица системы (1) имеет вид

$$
\left(\begin{array}{cc}
\xi_{1}+i \xi_{2} & -\xi_{3}-i \xi_{4} \\
\xi_{3}-i \xi_{4} & \xi_{1}-i \xi_{2}
\end{array}\right)
$$

отсутствие регуляризуемых краевых задач с граничными условиями «полиномиального типа относительно нормали» ранее установлено М.З. Соломяком [17]. Известен также результат В.С. Виноградова [18], что любая краевая задача типа задачи Римана-Гильберта не может быть нетеровской, если она ставится для эллиптической системы четырех дифференциальных уравнений первого порядка с действительными коэффициентами в $\mathrm{R}^{4}$, имеющей псевдосимметрический тип. Последний тип имеют, например, системы (9), если числа $b_{1} \quad$ и $b_{2}$ таковы, что $b_{1}^{2}+b_{2}^{2}=1$. Таким образом, теорема 3 обощает результат В.С. Виноградова.

3 а м е ч а н и е 4. Можно показать, хотя мы и не будем здесь останавливаться на этом, что добавление по методу Вишика-Эскина-Диканского ( см., например, [19] ) в граничные условия как для ЧКР-системы (1), так и для системы (9) любого конечного числа потенциалов с неизвестными плотностями по-прежнему не дает регуляризуемую краевую задачу. 


\section{БИБЛИОГРАФИЧЕСКИЙ СПИСОК}

1. Саак Э.М. К теории многомерных эллиптических систем первого порядка // Доклады АН СССР, 1975. Т. 222, № 1. C. 43-46.

2. Шевченко В.И. О задаче Гильберта для голоморфного вектора в многомерном пространстве // Дифференциальные и интегральные уравнения. Краевые задачи. Тбилиси, 1979. С.279-291.

3. Жадан М.И., Усс А.Т., Ющенко Д.П. Регуляризуемость краевых задач для одного класса многомерных аналогов системы Коши-Римана / Гомельск. гос. ун-т. Гомель, 1987. 17 с. Деп. в ВИНИТИ 01.04.87. № 2366-В87.

4. Балабаев B.E. Нормальные эллиптические системы первого порядка // Дифференциальные уравнения, 1995. Т. 31, № 1. C. 48-51.

5. Виноградов В.С. Об аналоге интеграла типа Коши для аналитических функций многих комплексных переменных // Доклады АН СССР, 1968. Т. 178, № 2. С. 282-285.

6. Виноградов В.С. О задаче Дирихле для многомерных эллиптических систем второго порядка // Доклады АН СССР, 1968. Т. 179, № 4. С. 766-767.

7. Янушаускас А.И. О некоторых системах с частными производными, связанных с многомерными аналогами системы Коши-Римана // Дифференциальные уравнения и их применения. Вильнюс, 1980. Вып. 27. С. 115-139.

8. Стейн И., Вейс Г. Введение в гармонический анализ на евклидовых пространствах. М.: Мир, 1974. 336 с.

9. Шевалле К. Теория групп Ли. Т. 1. М.: ГИИТЛ, 1948. 316 с.

10. Александров П.С. Комбинаторная топология. М.-Л.: ГОСТЕХИЗДАТ, 1947. 660 с.

11. Шевченко В.И. О гомотопической классификации многомерных эллиптических систем с комплексными коэффициентами // Доклады АН БССР, 1978. Т. 22, № 8. С. 681-683.

12. Самойленко И.С. Гомотопическая классификация систем двух псевдодифференциальных уравнений первого порядка и краевые задачи // Краевые задачи для уравнений в частных производных: Сборн. научн. трудов. Киев, 1978. C. 96-107.

13. Красносельский М.А., Забрейко П.П. Геометрические методы нелинейного анализа. М.: Наука, 1975. 512 с.

14. Агранович М.С. Эллиптические сингулярные интегро-дифференциальные операторы // Успехи математических наук, 1965. Т. 20, вып. 5. С. 3-120.

15. Лопатинский Я.Б. Об одном способе приведения граничных задач для систем дифференциальных уравнений эллиптического типа к регулярным интегральным уравнениям // Украинский математический журнал, 1953. Т. 5, № 2. C. $123-151$.

16. Волевич Л.Р. Разрешимость краевых задач для общих эллиптических систем // Математический сборник, 1965. T. 68, № 3. С. 373-416.

17. Соломяк М.3. О линейных эллиптических системах первого порядка // Доклады АН СССР, 1963. Т. 150 , № 1. C. 48-51.

18. Виноградов В.С. Граничная задача для псевдосимметрических систем // Дифференциальные уравнения, 1985. Т. 21, № 1. С. 161-163.

19. Эскин Г.И. Краевые задачи для эллиптических псевдодифференциальных уравнений. М.: Наука, 1973. 232 с. 